\title{
Maintenance and development of Symbiota2, a platform for data sharing and visualization
}

\author{
Mary Barkworth $\ddagger$, Andrew N. Miller§, Curtis Dyreson ${ }^{\ddagger}$, Benjamin Brandt!, William D. Pearse ${ }^{\ddagger}$ \\ ‡ Utah State University, Logan, United States of America \\ § University of Illinois Urbana-Champaign, Champaign, United States of America \\ | Northern Arizona University, Flagstaff, United States of America
}

Corresponding author: Mary Barkworth (mary.barkworth@usu.edu)

Received: 11 Aug 2017| Published: 14 Aug 2017

Citation: Barkworth M, Miller A, Dyreson C, Brandt B, Pearse W (2017) Maintenance and development of

Symbiota2, a platform for data sharing and visualization. Proceedings of TDWG 1: e20220.

https://doi.org/10.3897/tdwgproceedings.1.20220

\section{Abstract}

Symbiota is a database management system for aggregating and displaying record-based biodiversity information from collections of widely varying sizes and integrating them with images of living organisms and image-based records. It is currently used by over 230 collections that collectively provide access to records of over 20 million specimens. Its popularity is attributable to the low financial and learning barriers to participation in a Symbiota network and the wide array of tools it offers for creating resources needed by different user groups. It has been developed through grants, contracts, and pro bono contributions but it suffers from a limited pool of developers (for a project of its size), lack of a coordinated training program, and absence of a structure creating and expanded funding base for maintenance of the program and the networks that adopt it. Symbiota2 is designed to address these issues but development of a business plan is in its preliminary stages. It requires articulation of an overall vision for development of the program, knowledge of its current and potential user base, critical funding needs, and identification of those it benefits, particularly those capable of providing financial support. This presentation was developed in response to an invitation to speak at the Symposium. It outlines our preliminary thoughts on the subject. 
One of the first needs is to establish an entity, provisionally the Symbiota2 Foundation (S2F), to speak for Symbiota2. Its primary task would be fundraising. S2F would have an advisory board comprising individuals willing to help support Symbiota2 development, both personally and by promoting it to their contacts, plus representatives of Symbiota2's developers, networks, and network users. The first goal of the Foundation would be raise the funds required to ensure that any established networks remain active and secure by maintaining the currency of network installations, responding to bug reports, informing network managers of changes to the program, and making minor enhancements as requested by users. Grant funding will still be needed to add significant new features. Enhancements requiring major changes in the underlying program or development of completely new apps would still require grant or contract support. Before launching S2F, we need to build more effective communication with network and collection managers; create flyers, booklets, and presentations explaining the power of the program and its benefits to many different user groups; develop case studies illustrating benefits derived from using the program; and create at least one monetized app. These resources will make clearer the benefits that the biodiversity sciences, particularly Symbiota2, provide for the public because, ultimately, it is public support that is needed. We must use all the tools at our disposal to encourage people's interest in the organisms around them if we are to maintain support for biodiversity science and the collections on which it is based. Symbiota2 can play an important role in this regard. It is, however, unrealistic to expect that Symbiota2 will be able to develop the basic funds needed solely from monetized apps; it is also unrealistic to think it can be supported by charging data providers because many of them are, at present, unfunded. Consequently, there is no choice. We need to look initiate a fund raising program.

\section{Keywords}

Symbiota2, support, funding considerations, biodiversity record sharing software

\section{Presenting author}

Mary E. Barkworth 